\title{
Dynamic change in natural killer cell type in the human ocular mucosa in situ as means of immune evasion by adenovirus infection
}

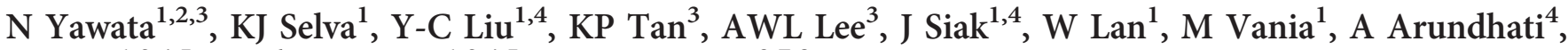

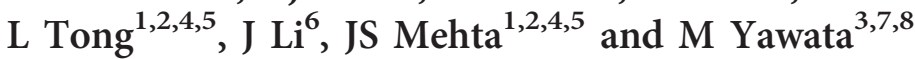

The most severe form of virus-induced inflammation at the ocular surface is epidemic keratoconjunctivitis (EKC), often caused by group D human adenoviruses (HAdVs). We investigated the dynamics and mechanisms of changes in natural killer (NK) cell types in the human ocular mucosal surface in situ over the course of infection. In the acute phase of infection, the mature CD56 ${ }^{\mathrm{dim}} \mathrm{NK}$ cells that comprise a major subpopulation in the normal human conjunctiva are replaced by CD56 ${ }^{\text {bright }} \mathrm{NK}$ cells recruited to the ocular surface by chemokines produced by the infected epithelium, and NKG2A-expressing CD56 ${ }^{\text {dim }}$ and CD56 ${ }^{\text {bright }}$ NK cells become the major subpopulations in severe inflammation. These NK cells attracted to the mucosal surface are however incapable of mounting a strong antiviral response because of upregulation of the inhibitory ligand human leukocyte antigen-E (HLA-E) on infected epithelium. Furthermore, group D HAdVs downregulate ligands for activating NK cell receptors, thus rendering even the mature NKG2A ${ }^{-}$NK cells unresponsive, an immune-escape mechanism distinct from other adenoviruses. Our findings imply that the EKC-causing group D HAdVs utilize these multiple pathways to inhibit antiviral NK cell responses in the initial stages of the infection.

\section{INTRODUCTION}

The conjunctiva is a mucosal surface that is a primary site of viral infection. Human adenoviruses (HAdVs) are the most frequent cause of acute viral conjunctivitis worldwide. ${ }^{1}$ Among the seven groups of human HAdVs $(A-G)$, group D viruses (e.g., HAdV-8, -19, and -37) cause severe, prolonged inflammation involving the cornea (epidemic keratoconjunctivitis (EKC)), whereas conjunctivitis caused by other HAdV groups are self-limiting and the inflammation is mild. ${ }^{2-4}$ Why the degree of ocular inflammation differs by adenoviruses is yet unclear.

Natural killer (NK) cells are one of the first lymphocytes to respond to viral infection. ${ }^{5}$ They are also key in promoting and regulating adaptive immunity through cytokine production and crosstalk with dendritic cells and T cells. ${ }^{6-8}$ The human NK cell population includes the immature $\mathrm{CD} 56^{\text {bright }}$, and mature CD56 ${ }^{\text {dim }}$ subpopulations, each with distinct profiles of inhibitory receptor expression and function., ${ }^{9,10}$ NK cell subpopulations vary by organ: $\mathrm{CD} 56^{\mathrm{dim}} \mathrm{NK}$ cells are dominant in peripheral blood, whereas $\mathrm{CD} 56^{\text {bright }} \mathrm{NK}$ cells inhabit many peripheral organs such as the liver and tonsil. ${ }^{11-13}$ Inhibitory NK cell receptors, such as members of the killer-cell immunoglobulin-like receptor (KIR) and NKG2A, recognize subsets of human leukocyte antigen (HLA)-A/B/C and HLA-E respectively, and are important in NK cell regulation. Notably, CD56 ${ }^{\text {bright }}$ NK cells express NKG2A almost exclusively with marginal KIR expression, whereas subsets of CD $56^{\mathrm{dim}} \mathrm{NK}$ cells express NKG2A and KIR heterogeneously. ${ }^{14,15}$

The profiles and function of human NK cells present within mucosal surfaces during viral infection is underexplored. In this

\footnotetext{
${ }^{1}$ Singapore Eye Research Institute, Singapore, Singapore. ${ }^{2}$ Duke-NUS Graduate Medical School, Clinical Sciences, Singapore, Singapore. ${ }^{3}$ Singapore Institute for Clinical Sciences (SICS), Agency for Science, Technology and Research (A*STAR), Singapore, Singapore. ${ }^{4}$ Singapore National Eye Centre, Singapore, Singapore. ${ }^{5}$ Department of Ophthalmology, Yong Loon Lin School of Medicine, National University of Singapore, Singapore, Singapore. ${ }^{6}$ Xinhua Hospital, Shanghai Jiao Tong University School of Medicine, Shanghai, China. ${ }^{7}$ Department of Pediatrics, Yong Loon Lin School of Medicine, National University of Singapore, Singapore, Singapore and ${ }^{8} \mathrm{National}$ University Health System, Singapore, Singapore. Correspondence: N Yawata (nobuyo.yawata@seri.com.sg)
} 
study, we characterized the human NK cells present in the conjunctiva and examined the cellular profiles during HAdV infection. We found that CD $56^{\mathrm{dim}} \mathrm{NK}$ cells are dominant in the healthy conjunctiva, and that the CD56 ${ }^{\text {bright }} \mathrm{NK}$ cells increase in situ during the acute phase of viral infection. Our analyses infer that group D HAdVs escape from conjunctiva NK cell immune responses by modulating NK cell subpopulations and altering expression of ligands for activating NK cell receptors.

\section{RESULTS}

Normal conjunctiva NK cells display a mature phenotype and are functionally competent: replacement by an immature CD56 ${ }^{\text {bright }}$ subpopulation during viral conjunctivitis

We first investigated the leukocyte populations present within the healthy conjunctiva (Figure 1a-c). Similar to peripheral blood NK cells, the conjunctiva CD56 ${ }^{\mathrm{dim}}$ cells were the dominant NK cell subpopulation and expressed KIR and NKG2A heterogeneously (Figure 1a,d,e). The functional responses of conjunctiva NK cells were also similar to that of peripheral blood NK cells, where the main response was through interferon- $\gamma($ IFN- $\gamma)$ and tumor necrosis factor- $\alpha$ $(\mathrm{TNF}-\alpha)$ production upon stimulation with phorbol 12myristate 13-acetate/ionomycin, whereas interleukin (IL)-17, IL-22, and IL-10 responses were marginal (Figure 1f). Therefore, the main population of conjunctiva NK cells is mature and capable of producing IFN- $\gamma$, a key cytokine in viral infection.

We examined the dynamics of conjunctiva leukocyte populations over the course of adenovirus infection. Leukocyte frequencies increased ninefold during the acute phase of conjunctivitis (Figure 2a,b), and NK cell and monocyte frequencies increased significantly (Figure $\mathbf{2 b}, \mathbf{c}$ ). Within the NK cell population, CD $56^{\text {bright }} \mathrm{NK}$ cells increased significantly, resulting in a 20 -fold elevation in the $\mathrm{CD} 56^{\text {bright }} / \mathrm{CD} 56^{\mathrm{dim}}$ ratio (0.88 in the acute phase of conjunctivitis and 0.044 in healthy conjunctiva; Figure 2a,d). In the convalescent phase, subpopulation profiles returned to one that resembled the healthy conjunctiva where $\mathrm{CD} 56^{\mathrm{dim}} \mathrm{NK}$ cells are the major subpopulation.

To probe the mechanisms underlying the significant increase in $\mathrm{CD} 56^{\text {bright }} \mathrm{NK}$ cells during acute viral conjunctivitis, the
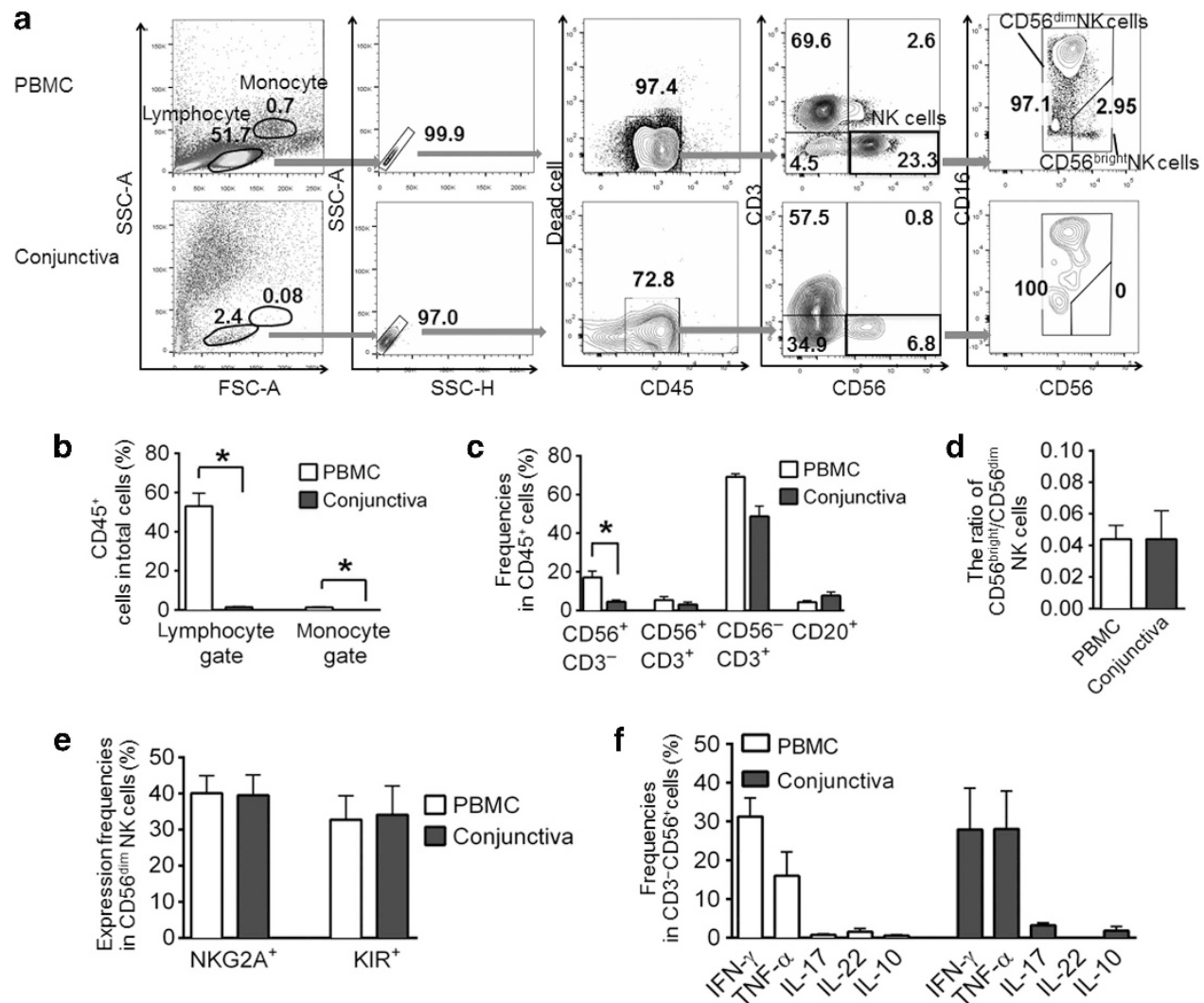

Figure 1 Conjunctiva natural killer (NK) cells are similar to those of peripheral blood NK cells. Leukocyte populations and NK cell profiles in conjunctiva brush cytology samples from healthy donors were compared with peripheral blood mononuclear cells (PBMCs). (a) Identification of leukocyte populations in conjunctiva brush cytology samples. The leukocyte gates used those adopted from peripheral blood. (b-e) Leukocyte frequencies were compared between peripheral blood and healthy conjunctiva. PBMC: $n=4$, conjunctiva: $n=8$. (b) Frequencies of CD45 ${ }^{+}$leukocytes among all cells in conjunctival samples observed in the lymphocyte and monocyte gates. (c) Frequencies of CD56 ${ }^{+} \mathrm{CD}^{-}, \mathrm{CD}^{-} 6^{+} \mathrm{CD}^{+}, \mathrm{CD}^{+} 6^{-} \mathrm{CD}^{+}$, and $\mathrm{CD}^{+} 0^{+}$lymphocytes among CD45 ${ }^{+}$lymphocytes. (d) Ratio of CD56 ${ }^{\text {bright }}$ NK to CD56 ${ }^{\text {dim }}$ NK cells. (e) Frequencies of NKG2A and KIR2DL2/3/DS2 expression on CD56 ${ }^{\text {dim }}$ NK

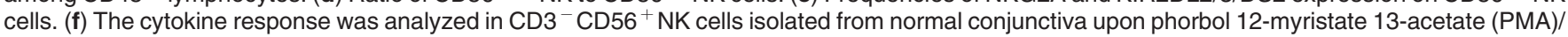
ionomycin stimulation and compared with that of peripheral blood NK cells. PBMC: $n=3$, conjunctiva: $n=3$. The mean + s.e.m. are shown. ${ }^{\star} P<0.05$. IFN- $\gamma$, interferon- $\gamma$; IL, interleukin; TNF- $\alpha$, tumor necrosis factor- $\alpha$. 
a

PBMC

conjunctivitis

<Acute phase>

Severe conjunctivitis

<Acute phase>

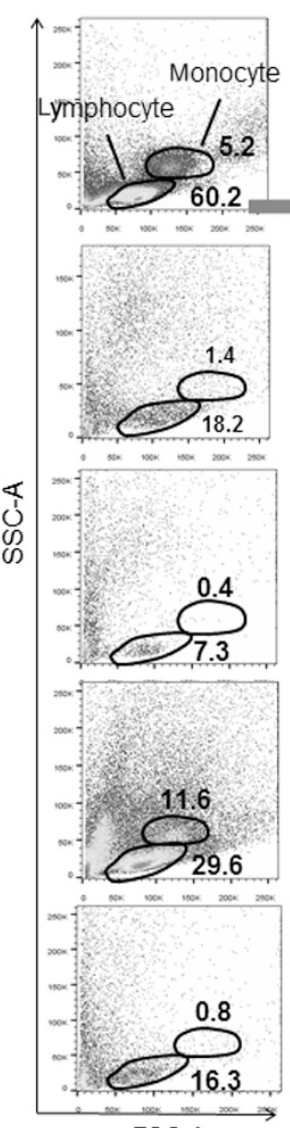

FSC-A
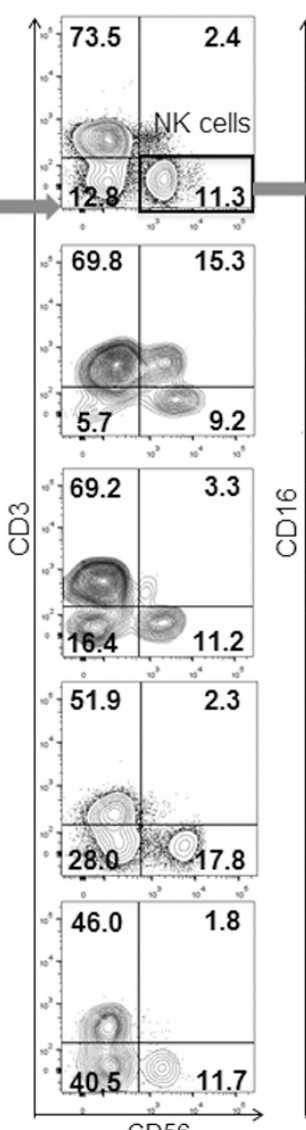

CD56

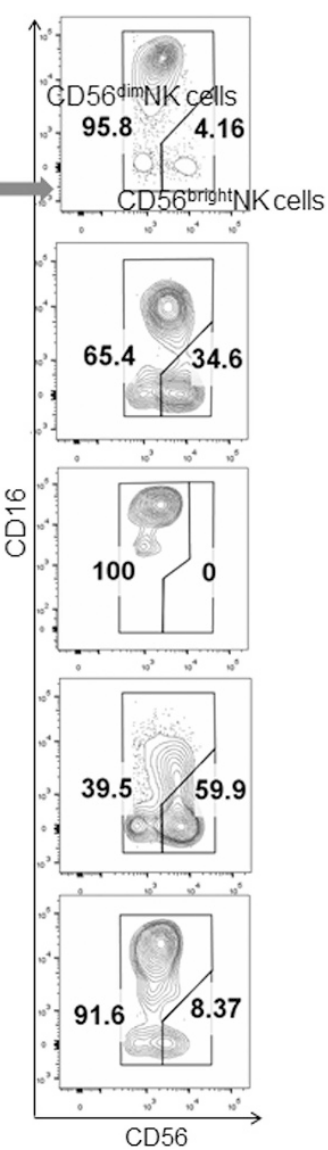

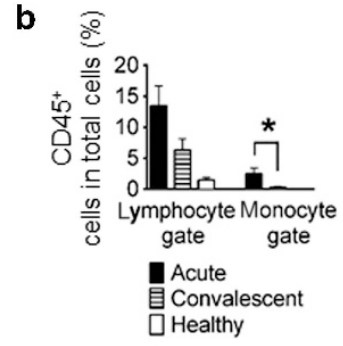

C
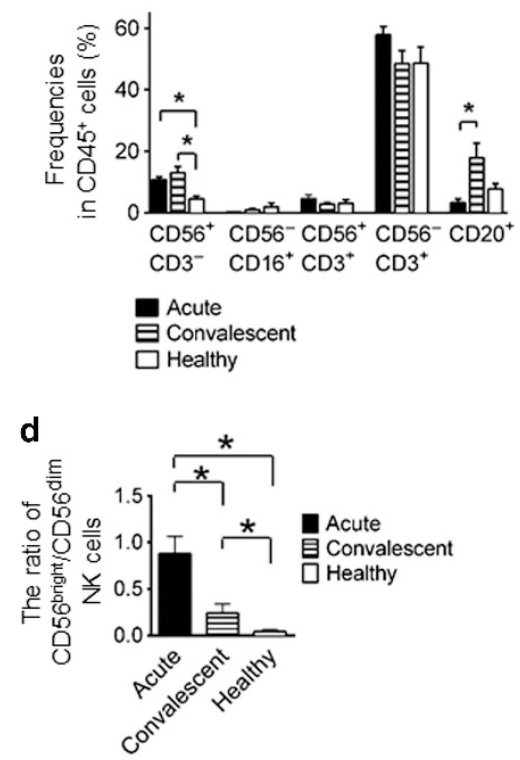

Figure 2 The conjunctiva CD56 $6^{\text {bright }} \mathrm{NK}$ cell population increases during acute viral conjunctivitis. (a) Representative profiles of cell populations in the conjunctiva of patients with adenovirus infection in the acute and convalescent phases in 12 eyes. (b-d) The frequencies of leukocytes in conjunctival samples from healthy donors and patients in the acute or convalescent phases of viral conjunctivitis were compared. Acute phase: $n=10$, convalescent phase: $n=12$, healthy control: $n=8$. (b) Frequencies of CD45 ${ }^{+}$leukocytes among all cells in the conjunctival samples. (c) Frequencies of CD56 ${ }^{+}$CD3 ${ }^{-}$,

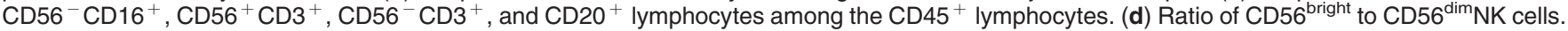
The mean + s.e.m. are shown. ${ }^{\star} P<0.05$. NK cell, natural killer cell; PBMC, peripheral blood mononuclear cell.

chemokines in tear fluid were analyzed using a multiplexed bead-based assay. CCL2 (CCR2 ligands), CCL3/4/5 (CCR5 ligands), and CXCL9/10 (CXCR3 ligands) were expressed at significantly higher levels in the acute phase (Figure 3a). These chemokines are known to recruit $\mathrm{CD} 56^{\text {bright }} \mathrm{NK}$ cells into inflammatory epithelia and synovial fluid through recognition by the receptors CXCR3, CCR2, and CCR5. ${ }^{16,17}$ Thus, we assessed expression of receptors for these chemokines on conjunctiva NK cells. CCR5 and CXCR 3 were highly expressed on both CD56 ${ }^{\text {bright }}$ and $\mathrm{CD} 56^{\mathrm{dim}}$ conjunctiva NK cells from all donors examined (Figure $\mathbf{3 b}, \mathbf{c}$ ). Expression of CCR2 was more variable among donors (Figure $\mathbf{3 b}$ ). In chemotaxis assays, we confirmed that $\mathrm{CD} 56^{\text {bright }} \mathrm{NK}$ cells migrated at significantly higher rates than $\mathrm{CD} 56^{\mathrm{dim}} \mathrm{NK}$ cells in the presence of the CCR5-binding chemokines CCL3/4/5 (Figure 3d,e).

We then assessed the activation status of conjunctiva NK cells during adenovirus infection by quantifying activation markers expressed on the NK cells. ${ }^{18-21}$ HLA-DR is suitable for this purpose as it is expressed stably for a long duration on activated lymphocytes. ${ }^{18,21}$ We found that NKG2A ${ }^{-} \mathrm{CD} 56^{\mathrm{dim}}$ conjunctiva NK cells were the only activated NK subset, and HLA-DR was not upregulated in the CD56 $6^{\text {bright }} \mathrm{NK}$ cells and $\mathrm{NKG}_{2} \mathrm{~A}^{+} \mathrm{CD} 56^{\mathrm{dim}} \mathrm{NK}$ cells (Figure 3f,g). CD38 expression was similar to that of HLA-DR where higher expression levels were observed on CD56 ${ }^{\mathrm{dim}}$ NK cells and CD56 ${ }^{\text {dim }} \mathrm{NKG}^{2} \mathrm{~A}^{-} \mathrm{NK}$ cells as compared with CD56 $6^{\text {bright }}$ and CD $56^{\mathrm{dim}} \mathrm{NKG}_{2 \mathrm{~A}}{ }^{+} \mathrm{NK}$ cells respectively (Figure $3 \mathbf{h}$, top panel). CD69 was upregulated constitutively on NK cells; however, NK cell subsets did not differ in expression levels (Figure $\mathbf{3 h}$, middle and bottom panels). The differences in activation marker profiles are likely because of their different modes of regulation, where secreted factors alone are sufficient to upregulate CD69 on NK cells, whereas cell-contact factors are known to be required for HLA-DR expression. ${ }^{20,22,23}$

\section{High expression of NKG2A on NK cells in severe inflammation}

We next sought to determine whether soluble factor production in conjunctival mucosa differs by the severity of inflammation. We found significantly elevated levels of CCL3/4/5 and 

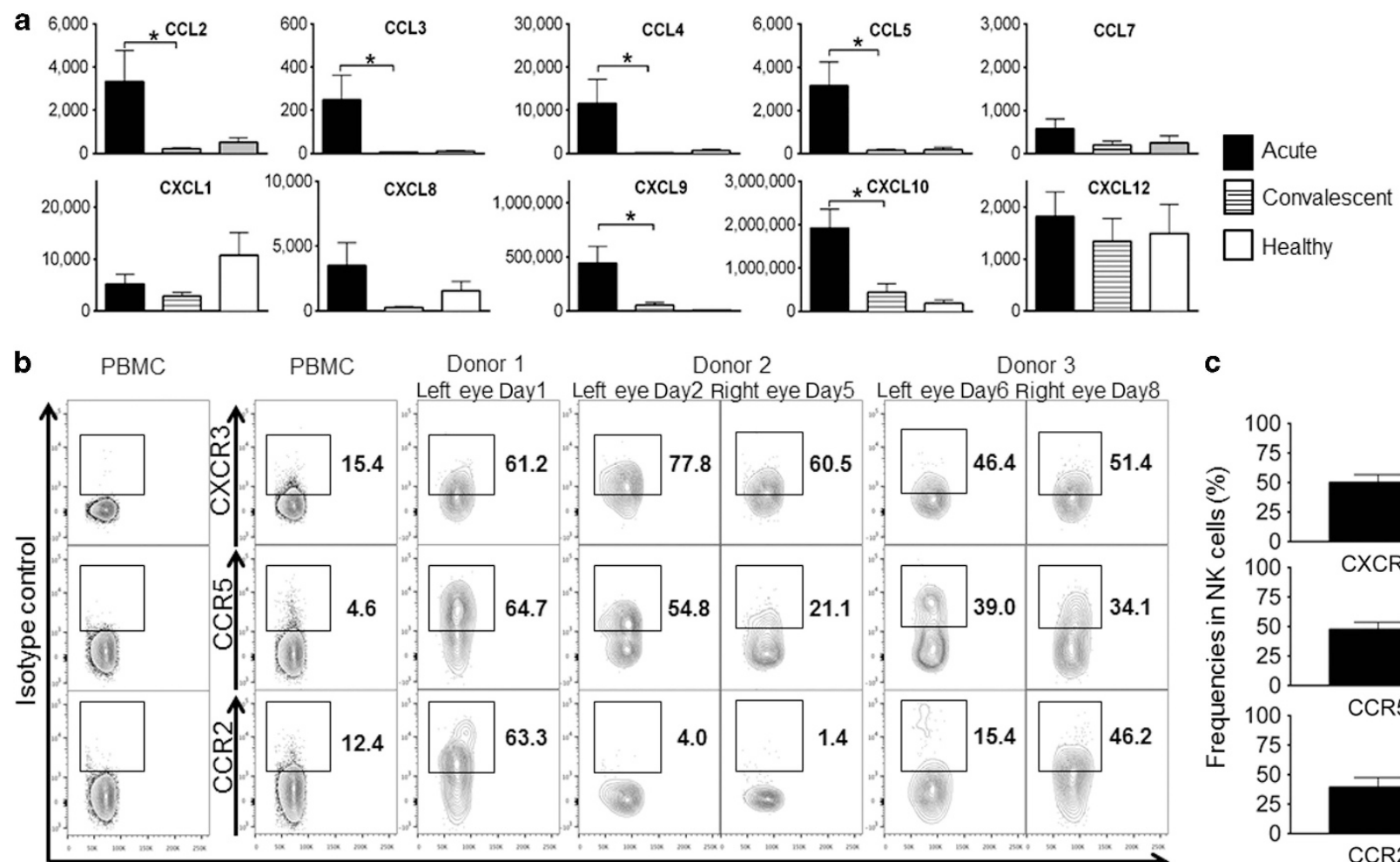

PBMC Donor 1

Donor 2

Donor 3

c Left eye Day1 Left eye Day2 Right eye Day5 Left eye Day6 Right eye Day8
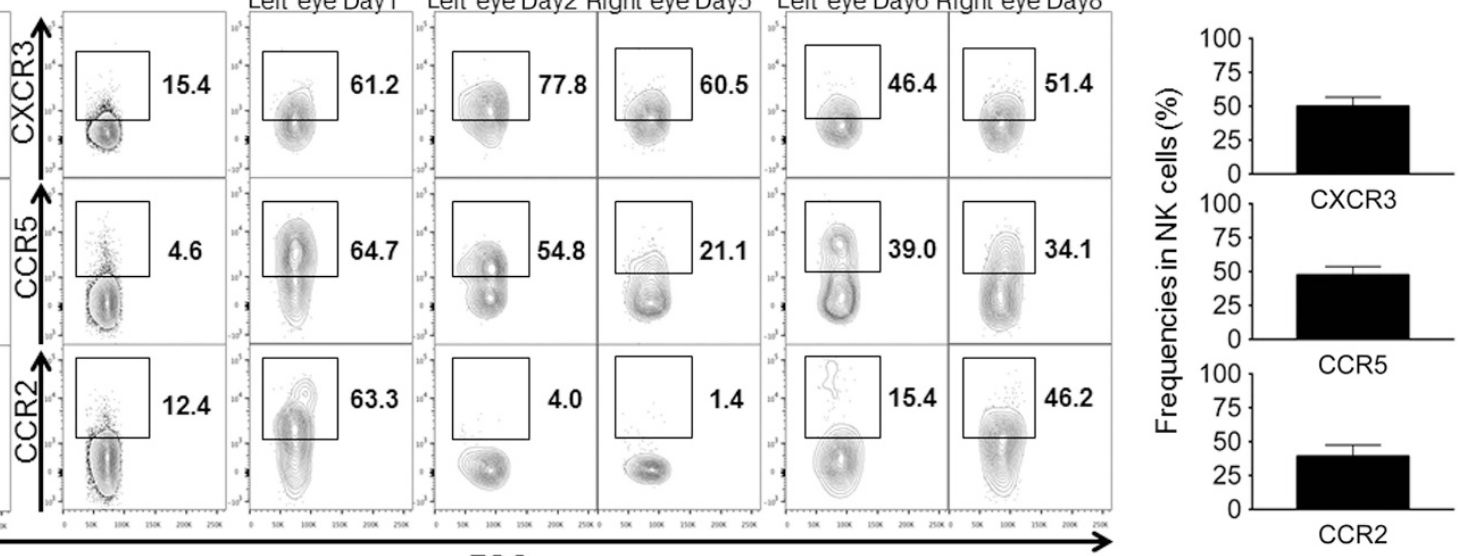

d

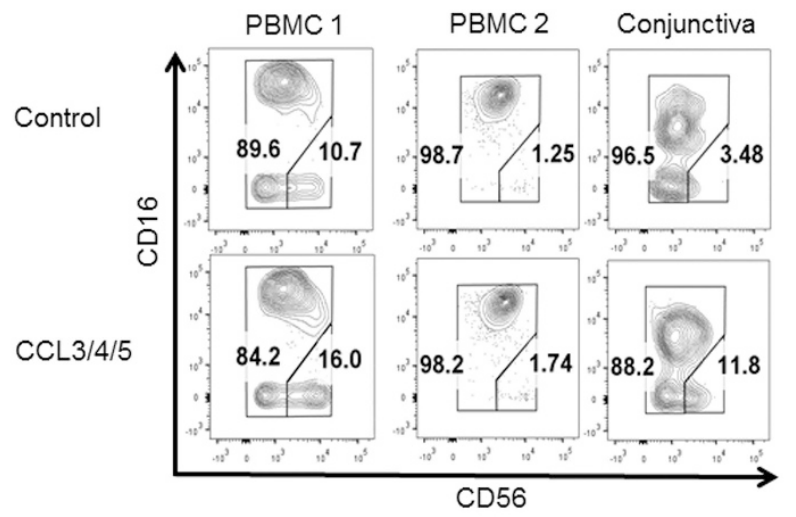

e

h
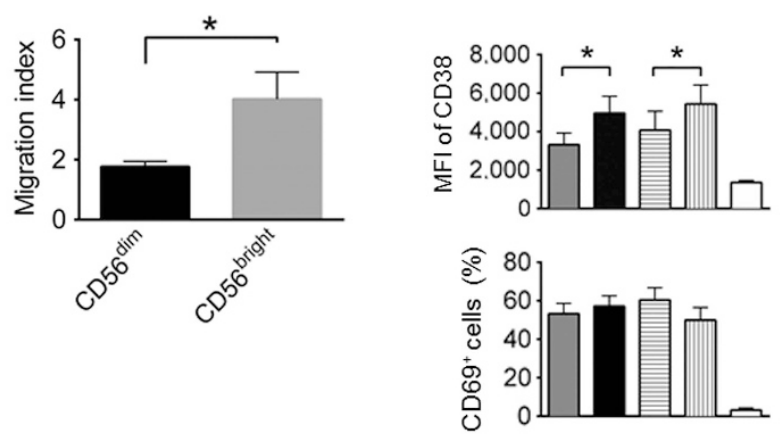

f

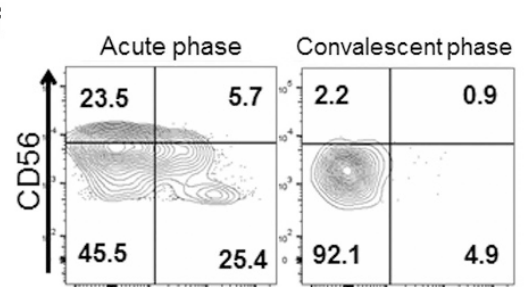

g
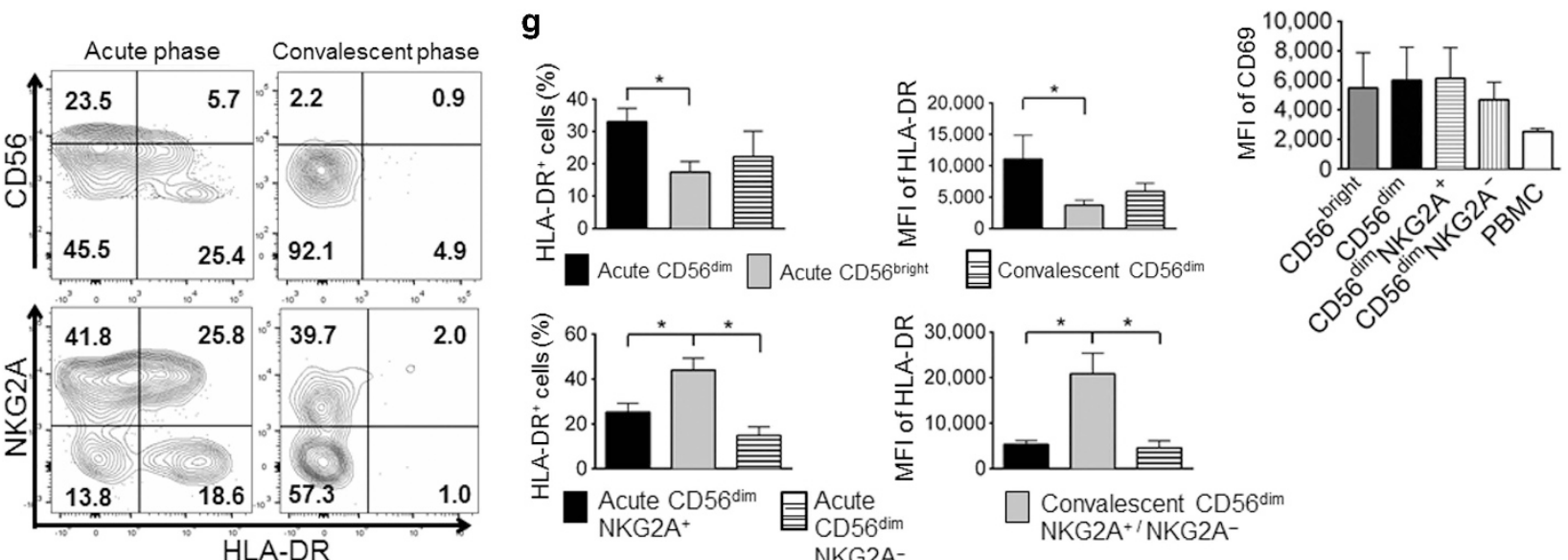
CCL2/CCL7 (ligands for CCR2) in severe inflammation, whereas no difference was observed for CXCL9/10 levels (Figure 4a). There was no significant difference in proinflammatory cytokine levels between severe and mild cases except for the IL-1 $\beta /$ IL-1ra ratio, and the main changes in cytokine profiles were those associated with acute conjunctivitis as compared with healthy or convalescent phase cases (Figure 4b).

We then investigated whether NK cell populations in conjunctival mucosa differ by severity of inflammation
(Figure 5). Although NK cells significantly increased in both severe and mild conjunctivitis, there was no difference in the overall frequencies of NK cells by severity. A distinct feature of severe conjunctivitis was the high frequency of NK cells expressing NKG2A. This was because of the significant increase in CD56 ${ }^{\mathrm{dim}} \mathrm{NK}$ cells expressing NKG2A, rather than an increase in CD56 ${ }^{\text {bright }} \mathrm{NK}$ cells whose frequencies were high in both severe and mild inflammation (Figure $\mathbf{5 b}, \mathbf{d}, \mathbf{e}$ ). We also noted that monocytes were present at a significantly
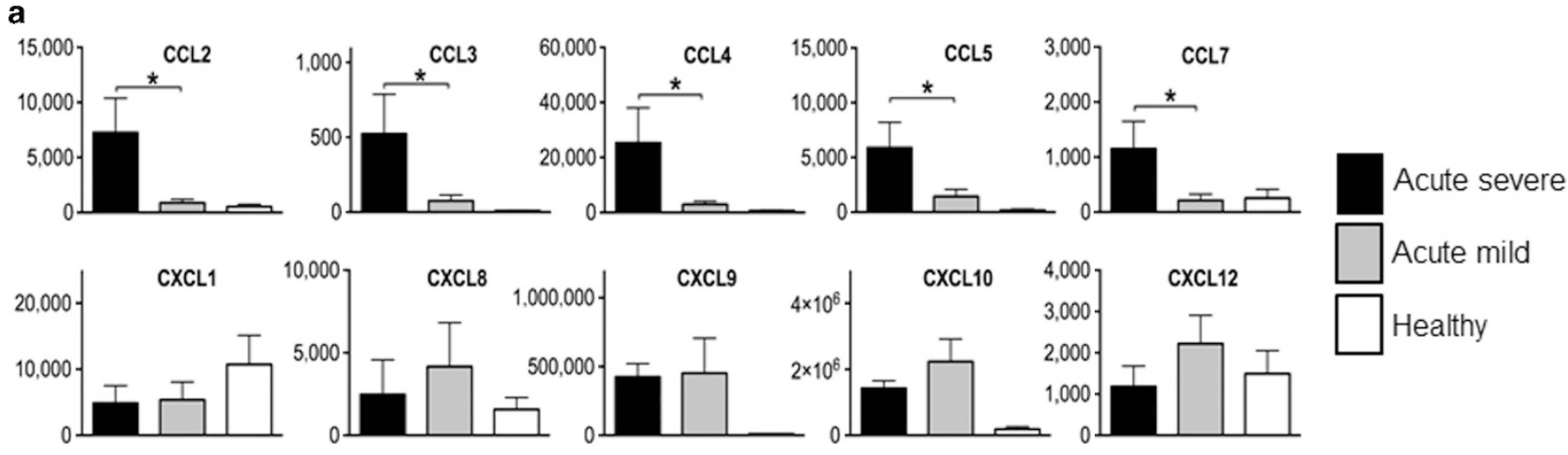

Acute mild
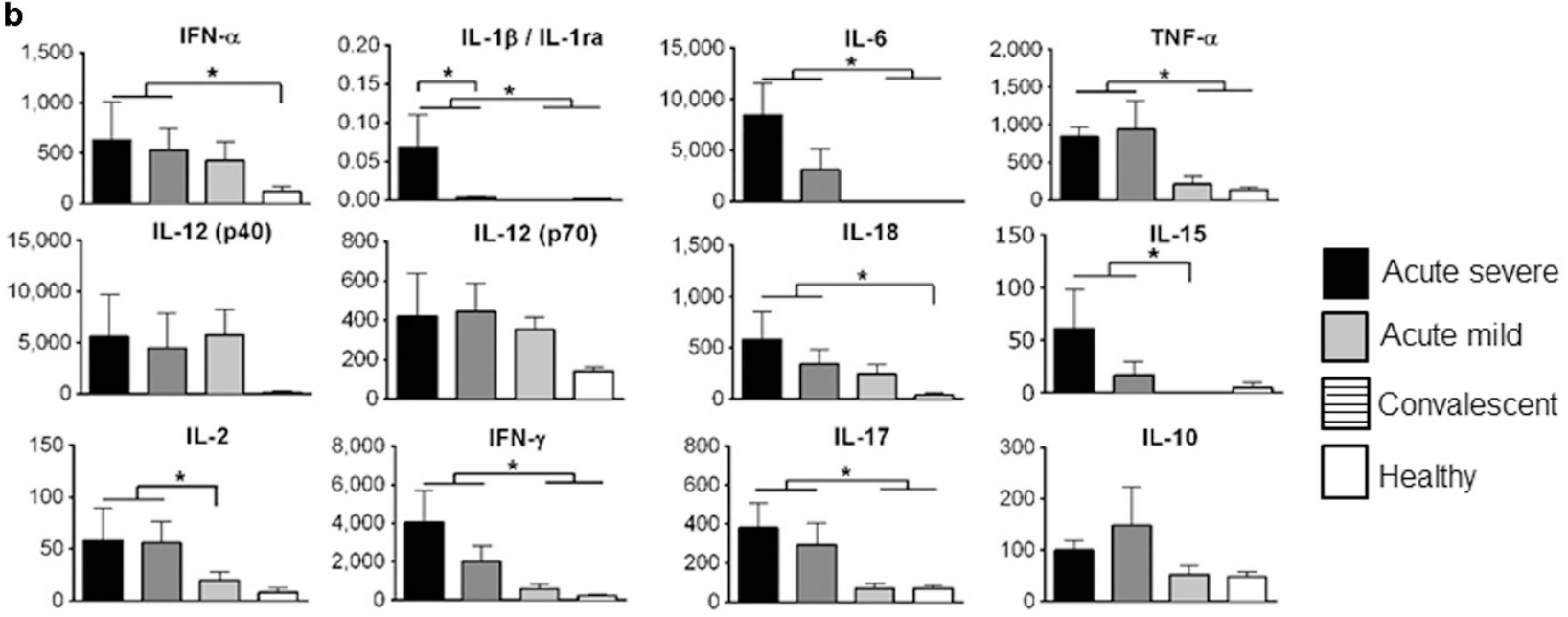

Figure 4 Upregulation of chemokines for CCR2/5 in tear fluid during severe inflammation. Tear fluid chemokine and cytokine profiles in acute-phase severe and mild conjunctivitis were analyzed using a multiplex beads assay. (a) Chemokines: acute severe conjunctivitis: $n=5$, acute mild conjunctivitis: $n=8$, and healthy control: $n=4$. (b) Cytokines: acute severe conjunctivitis: $n=5$, acute mild conjunctivitis: $n=8$, convalescent phase of conjunctivitis: $n=13$, and healthy control: $n=4$; data shown are in $\mathrm{pg} \mathrm{ml}^{-1}$. The mean + s.e.m. are shown. ${ }^{\star} P<0.05$. IFN, interferon; IL, interleukin; TNF, tumor necrosis factor.

Figure 3 Natural killer (NK) cells migrate to the ocular surface by CCR5/CXCR3 and display lower activation. (a) Tear fluid chemokine profiles in the acute and convalescent phases were analyzed using a multiplex beads assay; acute phase: $n=13$, convalescent phase: $n=13$, and healthy control: $n=4$. The data shown are in $\mathrm{p} \mathrm{ml}^{-1}$. (b) Representative expression of CXCR3, CCR5, and CCR2 on conjunctiva NK cells in the acute phase of viral conjunctivitis among 14 eyes. (c) Expression frequencies of CXCR3, CCR5, and CCR2 on the conjunctiva NK cells; $n=14$. (d) NK subset migration by CCL3/4/5 are compared. Shown are representative plots from three experiments. (e) Migration Indexes (the cell number that migrated in presence of the chemokines/the number of migrated cells with culture media) of CD56 ${ }^{\mathrm{dim}}$ and CD56 ${ }^{\text {bright }}$ NK cells are compared; $n=5$. (f) Representative flow cytometry plots comparing human leukocyte antigen-DR (HLA-DR) expression with CD56 expression on conjunctiva NK cells (upper two panels), and HLA-DR expression with NKG2A expression on CD56 ${ }^{\text {dim }}$ NK cells (lower two panels) in eight human adenovirus (HAdV) infection cases. (g) HLA-DR expression was compared between CD56 ${ }^{\text {dim }}$ and CD56 $6^{\text {bright }} \mathrm{NK}$ cells in the acute and convalescent phases of HAdV infection (upper two panels). Comparison of HLA-DR expression between NKG2A ${ }^{+}$and NKG2A ${ }^{-}$CD56 ${ }^{\text {dim }}$ NK cells (lower two panels). Acute phase of conjunctivitis: $n=8$, convalescent phase of conjunctivitis: $n=6$. (h) Mean fluorescence intensity (MFI) of CD38 (top panel), frequencies of CD69+ cells (middle panel), and MFI of CD69 (bottom panel) are compared between CD56 bright and CD56 ${ }^{\text {dim }} \mathrm{NK}$ cells, and NKG2A ${ }^{+}$and NKG2A ${ }^{-}$CD56 ${ }^{\text {dim }} \mathrm{NK}$ cells in acute conjunctivitis. CD38: $n=9$, CD69: $n=14$. The mean + s.e.m. are shown. ${ }^{*} P<0.05$. PBMC, peripheral blood mononuclear cells. 
higher frequency in the conjunctiva in severe conjunctivitis that was associated with increased levels of CCR2 ligands in the tear fluid of severe inflammation cases (Figures $4 \mathbf{a}$ and $5 \mathbf{a}$ ).
The NK cells licensed by KIR but not NKG2A are the main responding subsets in adenovirus infection

To understand the differences in NK cell profiles observed in conjunctivitis patients, we investigated the mechanisms by

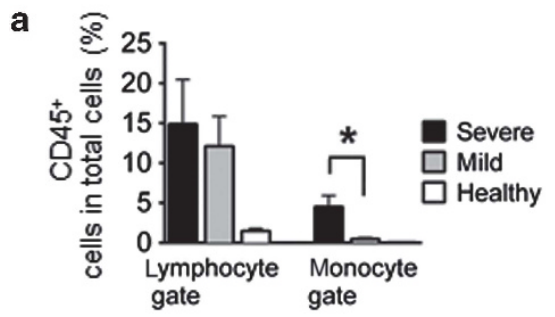

b
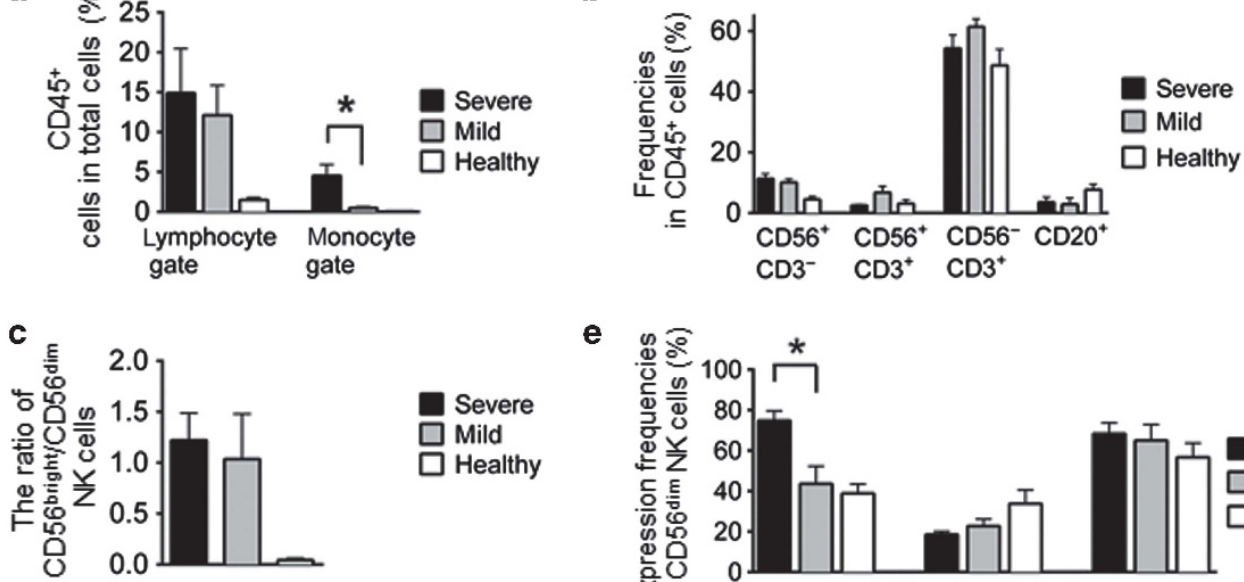

e

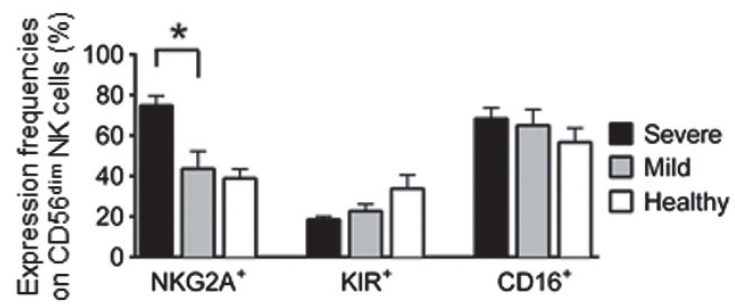

d
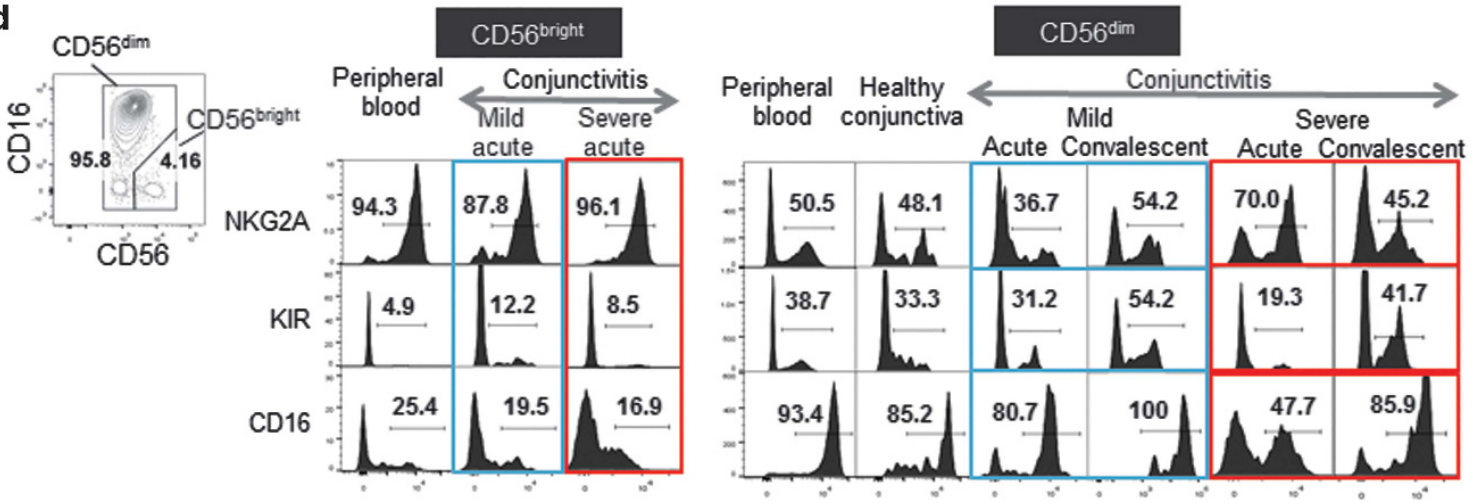

Figure 5 The $\mathrm{NKG}_{2} \mathrm{~A}^{+} \mathrm{NK}$ cell population increases in the conjunctiva during severe inflammation. (a) The frequencies of $\mathrm{CD}^{+} 5^{+}$cells in the total conjunctival samples from severe and mild conjunctivitis during the acute phase. (b) Frequencies of $\mathrm{CD} 56^{+} \mathrm{CD}^{-}, \mathrm{CD}^{-} 6^{+} \mathrm{CD}^{+}, \mathrm{CD}^{-} 6^{-} \mathrm{CD} 3^{+}$, and $\mathrm{CD} 20^{+}$lymphocytes among CD $45^{+}$cells. (c) Ratio of CD56 $6^{\text {bright }} \mathrm{NK}$ to CD56 ${ }^{\text {dim }}$ NK cells. (d) Representative histograms illustrating NKG2A, KIR2DL2/3/ S2, and CD16 expression on conjunctiva natural killer (NK) cells from 10 eyes with conjunctivitis. (d, left panels) Receptor expression on CD56 $6^{\text {bright }}$ NK cells from healthy peripheral blood and from acute mild (blue) and severe (red) conjunctivitis. (d, right panels) (from left) Receptor expression on CD56 ${ }^{\text {dim }}$ NK cells from healthy peripheral blood, healthy conjunctiva, and acute/convalescent phases of mild (blue) and severe (red) conjunctivitis. The numbers shown indicate the frequency of cells positive for each receptor. (e) Frequencies of NKG2A, KIR2DL2/3/DS2, and CD16 expression in CD56 ${ }^{\text {dim }}$ NK cells. Acute severe conjunctivitis: $n=5$, acute mild conjunctivitis: $n=5$, and healthy conjunctiva: $n=8$. The mean + s.e.m. are shown. ${ }^{*} P<0.05$.

Figure 6 Licensed/educated NKG2A ${ }^{-}$CD56 dim NK cells are the primary responders against adenovirus-infected cells. (a) Hexon protein expression in A549 cells and peripheral blood mononuclear cells (PBMCs) are shown. A549 epithelial cells were infected with human adenovirus (HAdV)-3 or HAdV-8. After 2 days, cells were coincubated with PBMCs from KIR ligand genotype-matched donors. (b) Representative flow cytometry plots for interferon- $\gamma$ $(\mathrm{IFN}-\gamma)$ production by $\mathrm{CD} 6^{+} \mathrm{CD}^{-}$NK cells among three donors as determined by intracellular staining after coincubation with mock-, HAdV-3-, or HAdV-8-infected cells. (c) Shown are IFN- $\gamma$-expressing NK cells after coincubation with cells infected with HAdV-3 and HAdV-8 in three donors. (d) IFN- $\gamma$ production by NK cells after coincubation with HAdV-3-infected cells compared with production by cells after replacement with fresh medium before coincubation, and by cells incubated with only the culture supernatant from infected cells. (e) Comparison of IFN- $\gamma$ production by NK cells after coincubation with HAdV-3-infected cells with and without culture-well insert. (f) Representative analyses of NK cell subset responses among three donors. IFN- $\gamma$ production by each of the $16 \mathrm{NK}$ cell subsets was determined by analyzing the expression of the four human leukocyte antigen (HLA) class I-specific inhibitory receptors involved in NK cell licensing/education: NKG2A, KIR2DL1, KIR2DL3, and KIR3DL1. ${ }^{14,26}$ The far left panel shows the combinations of receptors expressed on each NK cell subset (black boxes indicate receptor presence). Because the KIR ligands in the donors and in A549 were HLA-C1 and HLA-Bw4, the self-specific receptors are KIR2DL3, KIR3DL1, and NKG2A (underlined). Bar graphs (from left): NK subset responses against HAdV-3-infected cells, against HAdV-8-infected cells, against mock-infected cells, and against K562. (g) Comparison of IFN- $\gamma$ expression by NKG2A ${ }^{-}$and NKG2A ${ }^{+}$CD56 ${ }^{\text {dim }}$ NK cells responding to cells infected with HAdV-3 and HAdV-8 from three donors. (h) Expression of HLA class I and HLA-E on cells infected with HAdV-3 or HAdV-8 was analyzed 2 days after infection. Representative histograms from three experiments are shown. The mean + s.e.m. are shown. ${ }^{\star} P<0.05$. 
a

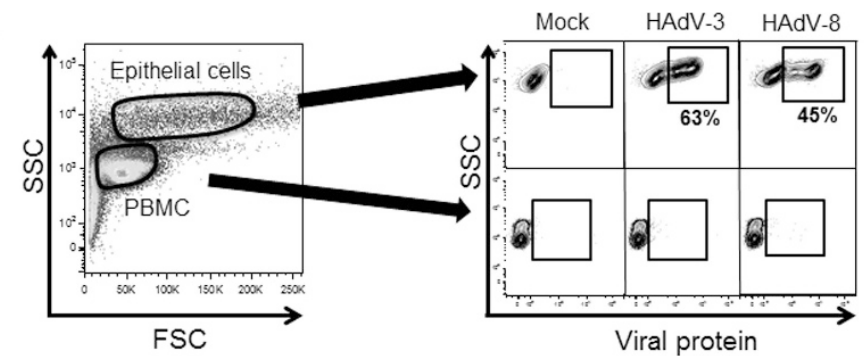

b

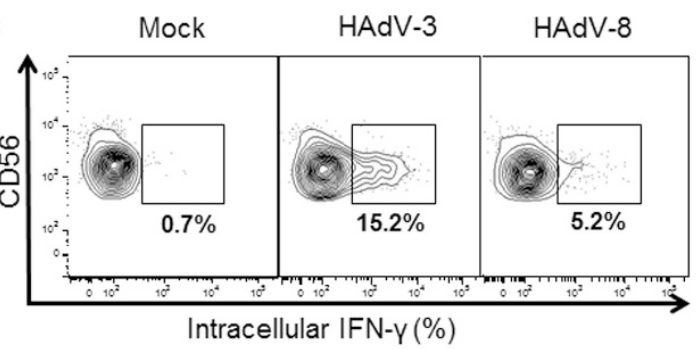

c

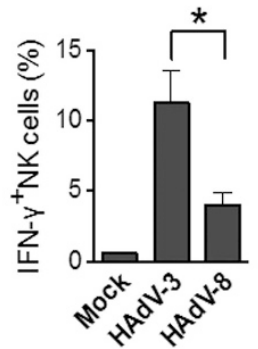

d

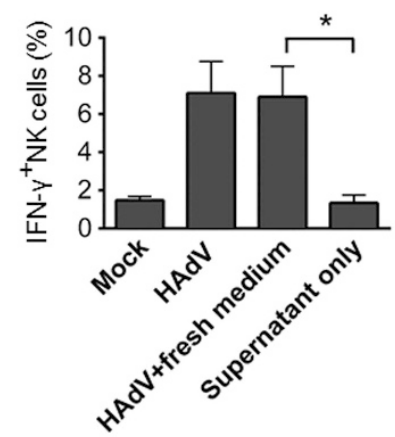

e

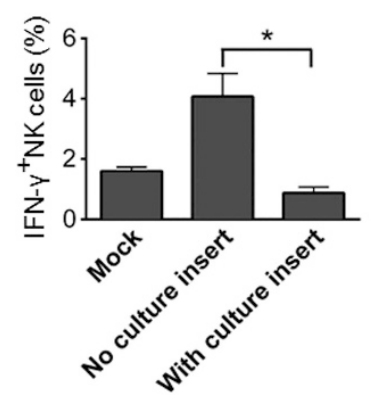

f

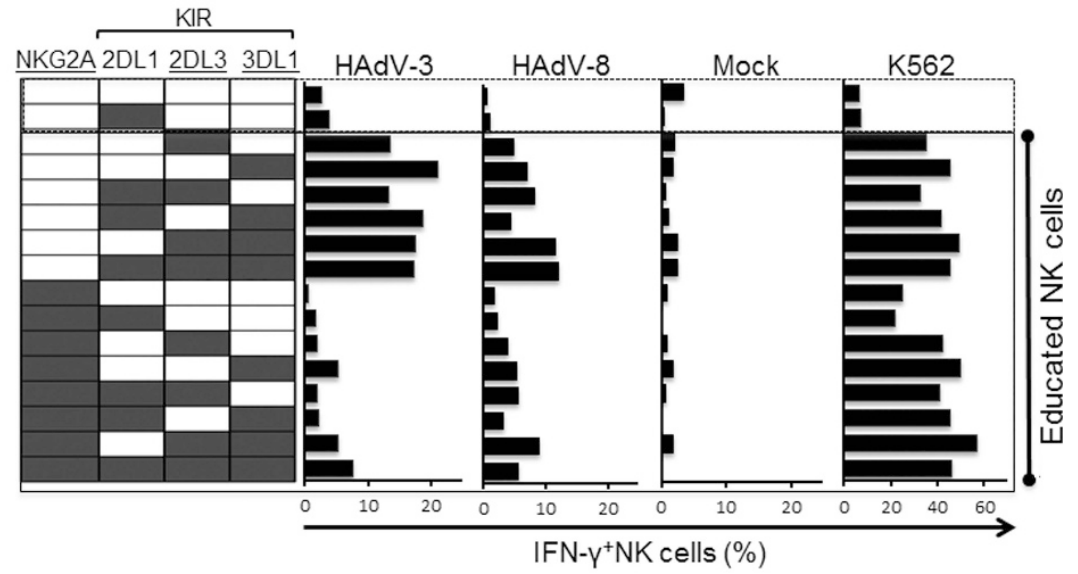

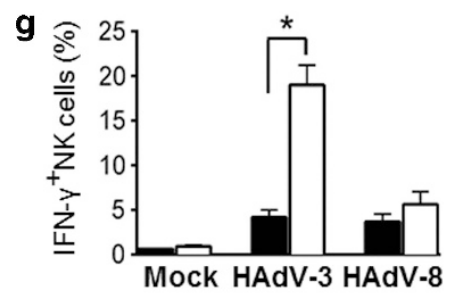

$\square$ NKG2A- $^{-}$

NKG2A+ h
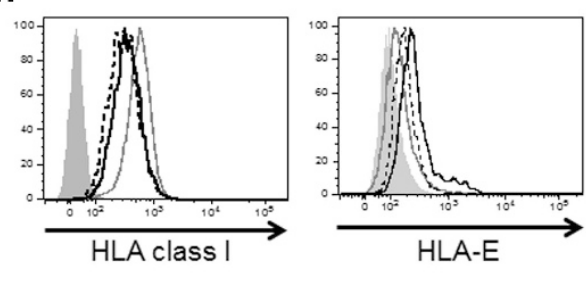

- Mock HAdV-3 ----. HAdV-8 
which NK cells respond against HAdV infection, using an in vitro coculture model. Using preoptimized virus titers, we infected mucosal epithelial cells (A549) with HAdV-3 (group B) and HAdV-8 (group D) viruses that cause mild and severe conjunctivitis respectively. Peripheral blood mononuclear cells (PBMCs) from healthy donors were coincubated with infected cells and NK cell cytokine responses were quantified by intracellular staining for IFN- $\gamma$. Notably, only CD56 ${ }^{\mathrm{dim}} \mathrm{NK}$ cells responded against the virus-infected cells and the IFN- $\gamma$ response was significantly stronger against HAdV-3-infected epithelial cells than HAdV-8-infected cells (Figure 6a-c). Importantly, direct cell contact with virus-infected cells was crucial for NK cell activation, where incubation with only the culture supernatant from HAdVinfected cells, or coincubation using a transwell system, abrogated NK cell responses (Figure 6d,e). In contrast, replacing the culture medium with fresh medium before coculture with PBMCs did not reduce the response. Therefore, we sought to identify the cell-contact factors involved in the NK cell response against HAdV. HAdVs are known to downregulate expression of HLA class I molecules on infected cells. ${ }^{24,25}$ NKG2A and KIR are involved in controlling the "missing-self" response; the mode of NK cell activation against cells with reduced HLA class I expression, and it is only the subsets of NK cells that express NKG2A or self-specific inhibitory KIR that are able to elicit a missing-self response (NK cell licensing/education). ${ }^{26}$ Upon comparing the responses of NKG2A- or KIR-expressing NK cell subsets against adenovirus-infected cells, the licensed/ educated NK cell subsets showed enhanced responses against HLA class I-deficient K562 cells as compared with nonlicensed/ noneducated NK cells (Figure 6f, bar graph on the right). Notably, only the licensed/educated $\mathrm{KIR}^{+} \mathrm{NK}$ cells, but not $\mathrm{NKG}_{2} \mathrm{~A}^{+} \mathrm{NK}$ cells, elicited strong responses against HAdVinfected cells (Figure 6f, bar graphs on the left). As a result, $\mathrm{NKG}_{2} \mathrm{~A}^{+} \mathrm{NK}$ cells displayed significantly lower responses against HAdV-3-infected cells than did $\mathrm{NKG}^{-} \mathrm{A}^{-}$cells (Figure 6g). A similar trend was observed in the responses against HAdV-8-infected cells, although the difference was smaller because of the weaker overall response against HAdV-8 infection.

An important question thus raised was whether the ligands for these inhibitory receptors are differentially modulated by HAdV infection. We observed that HAdV-3 and HAdV-8 downregulate HLA-A/B/C expression on infected cells at similar levels, whereas HLA-E, the ligand for the NKG2A receptor, is upregulated (Figure $\mathbf{6 h}$ ). The result is that HLA-E-mediated inhibition dampens the response of the $\mathrm{NKG} 2 \mathrm{~A}^{+} \mathrm{CD} 56^{\mathrm{dim}} \mathrm{NK}$ cell subsets and CD56 ${ }^{\text {bright }} \mathrm{NK}$ cells, and the NK cell response against HAdV-infected cells is limited to self-specific $\mathrm{KIR}^{+} \mathrm{CD} 56^{\mathrm{dim}} \mathrm{NK}$ cells that lack NKG2A expression, as assessed by HLA-DR and CD38 in the conjunctival samples.

The possibility of NK cell activation through NKG2C in the NKG2A ${ }^{-}$NK cells was investigated, as NKG2C is known to expand on NK cells in cytomegalovirus infection. ${ }^{27}$ This does not seem to be the case as we detected NKG2C expression in NK cells in only 3 out of the 12 conjunctival samples. Furthermore, NK cell responses did not differ between NKG2C-positive and negative NK cells (Supplementary Figure S1 online).

\section{Group D HAdVs impair NK cell responses by reducing expression of ligands for activating NK cell receptors}

Because NK cell responses were weaker against HAdV-8infected cells than infection by other HAdVs, we sought to identify other surface factors that could reduce NK cell responses. Here, we found that group D HAdVs dampen NK cell antiviral responses by downregulating ligand expression of the activating NK receptor DNAM-1.

Upon receptor blockade using antagonistic antibodies for NKG2D and DNAM-1 in coculture experiments with HAdVinfected epithelial cells, blocking either receptor nearly halved the NK cell response, and blocking both receptors totally abrogated the NK cell response to baseline level, inferring that these two activating receptors are the key factors that likely function synergistically in the anti-HAdV response (Figure 7a). We then analyzed the expression of ligands for these receptors on epithelial cells in vitro. The expression of both CD155 and CD112 (DNAM-1 ligands) on infected-A549 cells was substantially downregulated upon HAdV-8 infection (Figure $7 \mathbf{b}$ ) as compared with HAdV-3 infection. HAdV-3 infection however resulted in more upregulation of ULBP-1 and ULBP-2 (NKG2D ligands) as compared with HAdV-8 infection. These observations indicate that HAdV types differentially modulate the expression of ligands for NK cell receptors on infected cells.

To test whether a weak NK cell response is a common feature of group D HAdVs, we compared NK cell responses against cells infected with HAdV $-8,-10,-19 a$, and -37 (all group D) and cells infected with HAdV-3, -4 , and -5 (not group D) (Figure 7c). Only $1-4 \%$ of NK cells responded against group $\mathrm{D}$ virus-infected cells and these responses were significantly weaker than those against cells infected with group B and $\mathrm{E}$ HAdVs. This was consistent for prototype viruses obtained from ATCC (American Type Culture Collection) and clinically isolated virus strains. Furthermore, expression of CD155 and CD112 was significantly downregulated in the group D HAdVinfected cells as compared with cells infected with HAdVs not in group D (Figure 7d). These results infer that group D HAdVs share a distinct, common feature of dampening NK cell antiviral responses through downregulation of ligands for DNAM-1.

\section{DISCUSSION}

To the best of our knowledge, this is the first study detailing the dynamics of human NK cell profiles over the course of viral infection at a mucosal surface. Whereas mature CD56 ${ }^{\mathrm{dim}} \mathrm{NK}$ cells comprise a major $\mathrm{NK}$ cell population in the normal conjunctiva, CD56 ${ }^{\text {bright }} \mathrm{NK}$ cells accumulate in the tissue during the acute phase of adenovirus infection, as demonstrated by tear fluid chemokine profiles and the expression of the chemokine receptors CCR5 and CXCR3 on conjunctiva NK cells. Studies using animal models of virus infection, such as 


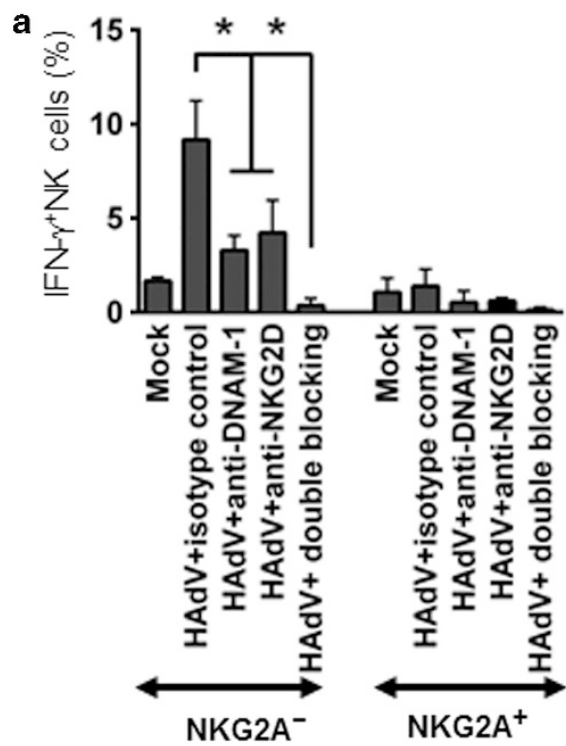

b
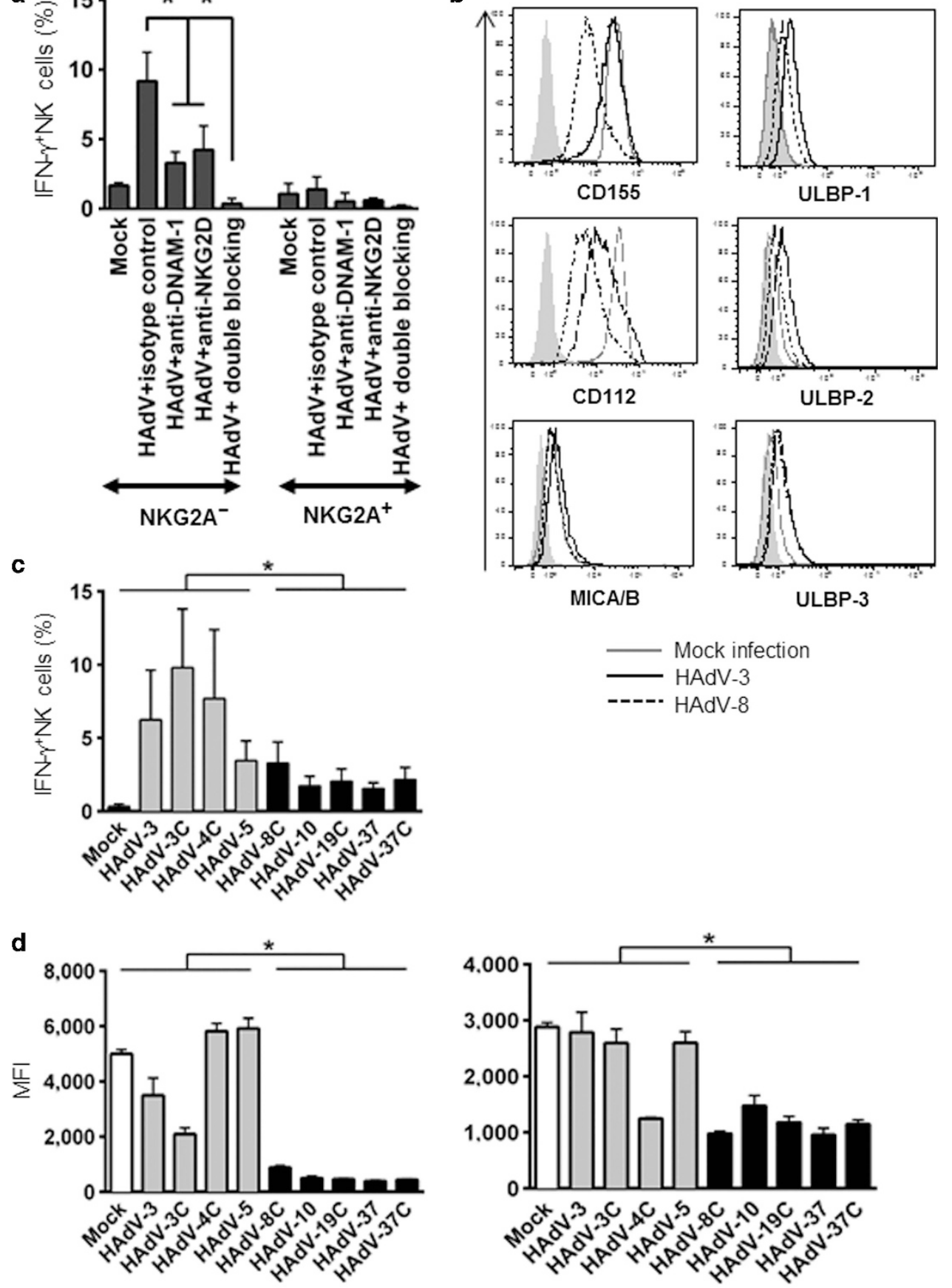

CD155

CD112

Figure 7 Group D human adenoviruses (HAdVs) impair natural killer (NK) cell responses by downregulating ligand expression of activating NK cell receptors. (a) Interferon- $\gamma$ (IFN- $\gamma$ ) production by NKG2A ${ }^{-}$NK cells and NKG2A ${ }^{+}$NK cells directed against HAdV-3-infected cells was analyzed in the presence of anti-DNAM-1 and/or anti-NKG2D blocking monoclonal antibodies. The data are results of NK cells from three donors. (b) Cell surface expression of ligands for DNAM-1 and NKG2D was analyzed on cells infected with HAdV-3 or HAdV-8. Representative histograms from three experiments are shown. (c) Comparison of the NK cell IFN- $\gamma$ responses against HAdV strains, including HAdV-3 (group B), -4 (group E), -5 (group C), -8 , $-10,-19$, and -37 (all group D) in three donors. "C" indicates HAdV strains isolated from clinical cases. (d) CD155 and CD112 expression in cells infected with different HAdV strains. Data are from three independent experiments (mean + s.e.m.). ${ }^{*} P<0.05$. MFI, mean fluorescence intensity.

herpes simplex virus infection, have indicated that these chemokine receptors are involved in NK cell trafficking to the ocular surface, lung, and genital mucosa. ${ }^{28-30}$

The drastic change in NK cell types effectively dampens the antiviral response in situ because the licensed, mature CD56 $6^{\mathrm{dim}}$
$\mathrm{NKG}_{2} \mathrm{~A}^{-} \mathrm{NK}$ cells that are capable of responding against adenovirus-infected epithelial cells are replaced by the hyporesponsive CD56 ${ }^{\text {bright }} \mathrm{NK}$ cells and NKG2A ${ }^{+} \mathrm{CD} 56^{\text {dim }} \mathrm{NK}$ cells. An important cell-contact factor affecting anti-HAdV NK cell responses is the upregulation of HLA-E and the 
downregulation of HLA-A/B/C expression. The consequence of this distinct profile in virus-induced modulation of HLA class I expression in infected epithelium is that the subsets responding to $\mathrm{HAdV}$ infection are limited to the minority fraction of NK cells licensed by KIR; all NKG2A-expressing subsets are inhibited by engagement with HLA-E, and the remaining unlicensed/uneducated subsets are intrinsically unable to mount a strong missing-self response. This mechanism agrees with previous studies that have described KIR-expressing mature CD56 ${ }^{\mathrm{dim}} \mathrm{NK}$ cells as the main responders in various virus infections, and highlights the importance of NK cell licensing/education in anti-virus NK responses. ${ }^{31,32}$

Downregulation of DNAM-1 ligands was identified as a second cell contact-based mechanism of NK cell response suppression as a means to escape the initial antiviral response mounted by conjunctiva-resident $\mathrm{CD} 56^{\mathrm{dim}} \mathrm{NK}$ cells. This mode of NK cell suppression is limited to infection by EKC-causing group D HAdVs. An insufficient initial NK cell response by cytokine production and cytotoxicity, and delayed clearance of the virus, will likely result in a prolonged innate proinflammatory cytokine response by monocytes and resident epithelial cells.

This study reveals that $\mathrm{CD} 56^{\mathrm{dim}} \mathrm{NK}$ cells are the dominant NK cell population in conjunctiva similar to those in peripheral blood, lung, and lower reproductive tract, and different from that described for other organs such as the liver, intestine, tonsil, and uterus where CD56 $6^{\text {bright }}$ NK cells are enriched. ${ }^{12,13,33,34}$ The CD56 ${ }^{\mathrm{dim}} \mathrm{NK}$ cells in the conjunctiva are mature and capable of producing IFN- $\gamma$ and TNF- $\alpha$, and thus are important as primary defense against infection at the ocular surface. The protection conferred is of importance to the ocular surface as well as the respiratory tract, because respiratory viruses such as avian influenza viruses and respiratory syncytial virus can also use the ocular surface as an initial site of entry and replication. ${ }^{35}$

Another important finding of our study was the elevated levels of IL-1 $\beta /$ IL-1ra and CCL2 in the tear fluid in severe conjunctiva inflammation. IL-1 $\beta$ is a critical ocular surface proinflammatory cytokine that is produced by corneal epithelial cells and monocytes, and the cytokine upregulates IL-6, IL-8, and CCL2 in conjunctiva epithelial cells and fibroblasts. ${ }^{36-38}$ IL-1 $\beta$ and CCL2 are factors that have been associated with severe inflammation and tissue damage in infectious diseases and in inflammatory diseases. ${ }^{39-42}$ Currently, corticosteroids are used to treat severe inflammation characteristic of EKC; however, more specific therapy such as IL-1 $\beta$ or IL-1 receptor blockade may be beneficial in curtailing excessive inflammation while enabling antiviral immune responses.

Understanding the dynamics of human NK cell populations and immune responses in the mucosal surface during viral infection is important for the development of new monoclonal antibody therapies and mucosal vaccines. Our study also demonstrates that the ocular surface is one anatomical site that may be ideal for investigations in human mucosal immunity.

\section{METHODS}

Human subjects. Patients with acute follicular conjunctivitis were recruited at the Singapore National Eye Centre and Changi General Hospital. The study was conducted in accordance with the Declaration of Helsinki Principles and was approved by the Singhealth Institutional Review Board. Written informed consent was taken from the participants. Tear fluid and conjunctival scrapings were collected in the acute and convalescent phases of EKC. Acute-phase samples were collected at the first visit (average 6.4 days, range 1-14 days after onset of symptoms). The severity of conjunctivitis was assessed using Efron grading, ${ }^{43}$ and conjunctival hyperemia of grade $\geq 3$ was classified as severe conjunctivitis. Patients were followed up over the course of conjunctivitis and the convalescent phase was defined as Efron grade $\leq 1$. Tear fluid was collected using Schirmer strip filter paper. ${ }^{44,45}$ Palpebral conjunctiva scrapings were obtained using the method of Fujihara et al. ${ }^{46,47}$ Adenovirus infection was diagnosed by assaying tear fluid for adenovirus genomic DNA, as described below. Patients who were treated with corticosteroid eye drops before the initial visit were excluded from the study. Samples collected from age-matched healthy donors served as non-HAdV controls. Cytokine production by conjunctiva NK cells was assessed using normal bulbar conjunctiva tissue obtained from patients who underwent pterygium excision at the Singapore National Eye Centre. Leukocytes were isolated from normal conjunctiva digested with $0.1 \mathrm{mg} \mathrm{ml}^{-1}$ of collagenase IV (Sigma-Aldrich, St Louis, MO). PBMCs from healthy donors were prepared by Ficoll gradient separation.

Viral DNA extraction and quantification. Tear fluid volume was estimated from the length of the wet portion of Schirmer strips using the following formula: tear volume $(\mu \mathrm{l})=0.62 \times$ length from the notch of paper $(\mathrm{mm})+0.54$. Immediately thereafter, Schirmer strips were soaked in phosphate-buffered saline containing $2.9 \% \mathrm{NaCl}$ and $0.5 \%$ Tween-20 for $4 \mathrm{~h}$ and the supernatant was collected. Viral genomic DNA was extracted using a Smitest EX-R\&D Nucleic Acid Extraction kit (Medical and Biological Laboratories, Nagoya, Japan). Hexon genespecific primers, as described by Miura-Ochiai et al., ${ }^{48}$ Power SYBR Green PCR Master Mix, and a PE7300 system (Applied Biosystems, Foster City, CA) were used for real-time PCR. PCR products were directly sequenced and used to confirm adenovirus infection.

Multiplex analysis of cytokines and chemokines in tear fluid. Cytokines and chemokines present in tear fluid were analyzed using a magnetic bead-based multiplex assay (Bio-Plex TM 200 System, Bio-Rad Laboratories, Hercules, CA). The analyses included probes for CCL2 (lower detection limit $\left.\left(\mathrm{pg} \mathrm{ml}^{-1}\right)=1.1\right)$, CCL3 (1.6), CCL4 (2.4), CCL5 (1.8), CCL7 (1.0), CXCL1 (6.3), CXCL8 (1.0), CXCL9 (1.2), CXCL10 (6.1), CXCL12 (8.7), IL-12 (p40) (23.3), IL-12 (p70) (3.5), IL18 (0.2), IL-2 (1.6), IL-15 (2.4), IFN- $\gamma$ (6.4), TNF- $\alpha$ (6.0), IL-1 $\beta$ (0.6), IL-1R $\alpha$ (5.5), IL-6 (2.6), IFN- $\alpha 2$ (4.3), IL-17 (3.3), and IL-10 (0.3). Calibration of the assay system and measurements followed the manufacturer's instructions.

Antibodies and flow cytometry. The following monoclonal antibodies were used: anti-CD56 (B159), anti-CD3 (SP34-2), antiKIR2DL2/3/2DS2 (CHL), anti-IFN- $\gamma$ (4S.B3), anti-IL-17 (N49-653), anti-IL-10 (JES3-19F1), anti-CD38 (HIT2), anti-CD69 (FN50) (BD Biosciences, San Jose, CA), anti-CD56 (MEM-188), antiKIR3DL1 (DX9), anti-KIR2DL1/2DS1 (HPMA4), anti-CD16 (3G8), anti-HLA class I (W6/32), anti-HLA-E (3D12), anti-HLA-DR (L243), anti-CD112 (TX31), anti-CD155 (SKII.4), anti-MICA/MICB (6D4), anti-TNF- $\alpha$ (MAb11), anti-CXCR3 (G025H7), anti-CCR2 (K036C2), anti-CCR5 (HEK/185a) (Biolegend, San Diego, CA), anti-CD45 (HI30) (Invitrogen, Carlsbad, CA), anti-KIR3DL1 (DX9) (Miltenyi Biotech, Bergisch Gladbach, Germany), anti-KIR2DL2/3/2DS2 (GL183), anti-NKG2A (Z199) (Beckman Coulter, Brea, CA), antiHLA-DR (LN3), anti-IL-22 (22URTI) (eBioscience, San Diego, CA), anti-ULBP1 (170818), anti-ULBP2 (165903), anti-ULBP3 (166510), and anti-NKG2C (134591) (R\&D Systems, Minneapolis, MN). Dead 
cells were excluded using LIVE/DEAD Fixable Dead Cell Staining kits (Invitrogen). Brush cytology samples were immediately soaked in RPMI-1640 medium supplemented with $10 \%$ heat-inactivated fetal calf serum (RPMI-10). For intracellular staining, cells were permeabilized with Cytofix/Cytoperm solution (BD Biosciences). Samples were analyzed by 13-parameter flow cytometry using an LSRII cell analyser (BD Biosciences). CD56 ${ }^{\mathrm{dim}}$ and CD56 ${ }^{\text {bright }} \mathrm{NK}$ cells were defined by the gating strategy described in Figure 1a, and the gates were applied in the analyses of all samples. Cytometry data were analyzed using FlowJo software (Tree Star, Ashland, OR).

Virus propagation and purification. HAdV $-3,-5,-10$, and -37 were obtained from ATCC (Manassas, VA). Clinical strains of HAdV-3, -4, $-8,-19 a$, and -37 were isolated from conjunctival samples from patients with adenovirus-associated conjunctivitis and were propagated in A549 using the methods described by Aoki et al. ${ }^{49}$ Propagated adenoviruses were purified using a Mini adenovirus purification kit (Biomiga, San Diego, CA) following the manufacturer's instructions. Purified viruses were titrated using an enzyme immunoassay.

Infection of epithelial cells and detection of viral protein. The day before infection, $1.8 \times 10^{4}$ A549 epithelial cells were seeded into a 96-well culture plate with Dulbecco's modified Eagle's medium/Ham's F12 media supplemented with $10 \%$ fetal calf serum. At $80 \%$ confluence, the cells were infected with purified HAdV in $30 \mu \mathrm{l}$ of Eagle's minimal essential medium supplemented with $2 \%$ fetal calf serum for $1 \mathrm{~h}$ by gently mixing every $15 \mathrm{~min}$ before addition of culture medium. The frequency of infection was assessed via intracellular staining for HAdV hexon protein using an anti-hexon antibody (AB1056F, Millipore, Billerica, MA) and subsequent flow cytometry analysis. The infection conditions were optimized to attain $30 \pm 10 \%$ of virus protein-positive cells after $48 \mathrm{~h}$ of infection (between 0.3 and 10 multiplicity of infection).

Functional analysis of conjunctiva NK cells. Conjunctival leukocytes were stimulated with $2 \mathrm{ng} \mathrm{ml}^{-1}$ of phorbol 12-myristate 13-acetate and $1 \mu \mathrm{g} \mathrm{ml}^{-1}$ of ionomycin (Sigma-Aldrich) in RPMI-10 at $37^{\circ} \mathrm{C}$ and $5 \% \mathrm{CO}_{2}$ for $5 \mathrm{~h}$. Brefeldin A (Sigma-Aldrich) and monensin (BD Biosciences) were added for the last $4 \mathrm{~h}$. To assess NK cell responses against $\mathrm{HAdV}$-infected cells, A549 cells preinfected with HAdV at predetermined multiplicity of infection were coincubated with PBMCs at a effector to target ratio of 10:1 in RPMI- 10 at $37^{\circ} \mathrm{C}$ and $5 \% \mathrm{CO}_{2}$ for a total of $16 \mathrm{~h}$ and brefeldin A was added $1 \mathrm{~h}$ after coculture. For blocking experiments, PBMCs were preincubated with purified monoclonal antibody (anti-NKG2D (1D11, Biolegend) and/or anti-DNAM-1 (102511, R\&D Systems)) or a corresponding isotype control at $10 \mu \mathrm{g} \mathrm{ml}^{-1}$ for $20 \mathrm{~min}$ before coincubation with A549 cells. To assess missing-self responses of NK cells, PBMCs were cocultured with HLA class I-deficient K562 cells at an effector to target ratio of 5:1 with $2,000 \mathrm{U} \mathrm{ml}^{-1}$ of IL-2 (R\&D Systems) for $14 \mathrm{~h}^{14}$

KIR genotyping and ligand typing. To avoid triggering allogeneic responses in the NK cells used in the coincubation assays, healthy donors with KIR ligands identical to those of A549 cells were screened and selected as a source for PBMCs. KIR ligand typing was performed using a KIR HLA-ligand SSP kit (Olerup, Stockholm, Sweden). KIR genotyping used the PCR-SSP KIR genotyping system described by Vilches et al. ${ }^{50}$ KIR genotypes of the three donors used for coincubation assay were group A haplotype homozygotes (presence of KIR2DL1/2DL3/3DL1/2DS4/3DL2, and absence of KIR2DL2, 2DS1, 2DS2, and 3DS1).

Chemotaxis assay. Cell migration was assessed using 24-well transwell inserts (Corning, NY). RPMI-1640 medium $(600 \mu \mathrm{l})$ supplemented with $0.5 \%$ bovine serum albumin was added to the lower wells, with/without $100 \mathrm{ng} \mathrm{ml}^{-1}$ of CCL3, CCL4, and CCL5 (R\&D Systems) as the chemoattractants. PBMCs from healthy donors and brush cytology samples from severe acute follicular conjunctivitis patients were each resuspended in $100 \mu \mathrm{l}$ of RPMI-1640 medium supplemented with $0.5 \%$ bovine serum albumin, and loaded onto the respective inserts. After $3 \mathrm{~h}$ of incubation, the inserts were removed and the cells in the lower wells were collected and analyzed by flow cytometry.

Statistical analysis. Data were statistically analyzed using Prism software (GraphPad Software, La Jolla, CA). Mann-Whitney tests were used to analyze patient samples, and $t$-tests were used for the in vitro analyses. $P$-values of $<0.05$ with a two-tailed test were considered significant.

SUPPLEMENTARY MATERIAL is linked to the online version of the paper at http://www.nature.com/mi

\section{ACKNOWLEDGMENTS}

We thank all donors, Abriena Xie for sample collection, Kenji Ohba, Hisatoshi Kaneko, and Tsuguto Fujimoto for technical advice on adenovirus propagation, and Koki Aoki, Shigeaki Ohno, Naoki Yamamoto, and Antonio Bertoletti for discussions. This work was supported by the Agency for Science, Technology and Research (A*STAR) and the National Medical Research Council (NMRC), Singapore.

\section{DISCLOSURE}

The authors declared no conflict of interest.

c) 2016 Society for Mucosal Immunology

\section{REFERENCES}

1. Gordon, Y. Ocular Infection and Immunity, 877-894 (Mosby Publishing, St Louis, 1996).

2. Aoki, K. \& Tagawa, Y. A twenty-one year surveillance of adenoviral conjunctivitis in Sapporo, Japan. Int. Ophthalmol. Clin. 42, 49-54 (2002).

3. Robinson, C.M., Seto, D., Jones, M.S., Dyer, D.W. \& Chodosh, J. Molecular evolution of human species D adenoviruses. Infect. Genet. Evol. 11, 1208-1217 (2011)

4. Nilsson, E.C. et al. The GD1a glycan is a cellular receptor for adenoviruses causing epidemic keratoconjunctivitis. Nat. Med. 17, 105-109 (2011).

5. Lodoen, M.B. \& Lanier, L.L. Natural killer cells as an initial defense against pathogens. Curr. Opin. Immunol. 18, 391-398 (2006).

6. Lanier, L.L. Evolutionary struggles between NK cells and viruses. Nat. Rev. Immunol. 8, 259-268 (2008).

7. Andrews, D.M. et al. Innate immunity defines the capacity of antiviral T cells to limit persistent infection. J. Exp. Med. 207, 1333-1343 (2010).

8. Andoniou, C.E., Coudert, J.D. \& Degli-Esposti, M.A. Killers and beyond: NK-cell-mediated control of immune responses. Eur. J. Immunol. 38, 2938-2942 (2008).

9. Cooper, M.A., Fehniger, T.A. \& Caligiuri, M.A. The biology of human natural killer-cell subsets. Trends Immunol. 22, 633-640 (2001).

10. Halama, N. et al. Natural killer cells are scarce in colorectal carcinoma tissue despite high levels of chemokines and cytokines. Clin. Cancer Res. 17, 678-689 (2011).

11. Sojka, D.K., Tian, Z. \& Yokoyama, W.M. Tissue-resident natural killer cells and their potential diversity. Semin. Immunol. 26, 127-131 (2014).

12. Carrega, P. et al. CD56(bright)perforin(low) noncytotoxic human NK cells are abundant in both healthy and neoplastic solid tissues and recirculate to secondary lymphoid organs via afferent lymph. J. Immunol. 192, 3805-3815 (2014).

13. Shi, F.D., Ljunggren, H.G., La Cava, A. \& Van Kaer, L. Organspecific features of natural killer cells. Nat. Rev. Immunol. 11, 658-671 (2011).

14. Yawata, M. et al. MHC class I-specific inhibitory receptors and their ligands structure diverse human NK-cell repertoires toward a balance of missing self-response. Blood 112, 2369-2380 (2008). 
15. Romagnani, C. et al. CD56brightCD16- killer Ig-like receptor- NK cells display longer telomeres and acquire features of CD56dim NK cells upon activation. J. Immunol. 178, 4947-4955 (2007).

16. Gregoire, C. et al. The trafficking of natural killer cells. Immunol. Rev. 220, 169-182 (2007).

17. de Matos, C.T. et al. Activating and inhibitory receptors on synovial fluid natural killer cells of arthritis patients: role of CD94/NKG2A in control of cytokine secretion. Immunology 122, 291-301 (2007).

18. Azeredo, E.L. et al. NK cells, displaying early activation, cytotoxicity and adhesion molecules, are associated with mild dengue disease. Clin. Exp. Immunol. 143, 345-356 (2006).

19. Zhang, Z. et al. Hypercytolytic activity of hepatic natural killer cells correlates with liver injury in chronic hepatitis B patients. Hepatology 53, 73-85 (2011).

20. Benlahrech, A. et al. Human NK cell up-regulation of CD69, HLA-DR, interferon gamma secretion and cytotoxic activity by plasmacytoid dendritic cells is regulated through overlapping but different pathways. Sensors (Basel) 9, 386-403 (2009).

21. Aranami, T., Miyake, S. \& Yamamura, T. Differential expression of CD11c by peripheral blood NK cells reflects temporal activity of multiple sclerosis. J. Immunol. 177, 5659-5667 (2006).

22. Draghi, M. etal. NKp46 and NKG2D recognition of infected dendritic cells is necessary for NK cell activation in the human response to influenza infection. J. Immunol. 178, 2688-2698 (2007).

23. Romagnani, C. et al. Activation of human NK cells by plasmacytoid dendritic cells and its modulation by CD4 + T helper cells and CD4 + CD25hi T regulatory cells. Eur. J. Immunol. 35, 2452-2458 (2005).

24. Fu, J., Li, L. \& Bouvier, M. Adenovirus E3-19K proteins of different serotypes and subgroups have similar, yet distinct, immunomodulatory functions toward major histocompatibility class I molecules. J. Biol. Chem. 286, 17631-17639 (2011).

25. Korner, H. \& Burgert, H.G. Down-regulation of HLA antigens by the adenovirus type 2 E3/19K protein in a T-lymphoma cell line. J. Virol. 68, 1442-1448 (1994).

26. Anfossi, N. et al. Human NK cell education by inhibitory receptors for MHC class I. Immunity 25, 331-342 (2006).

27. Lopez-Botet, M., Muntasell, A. \& Vilches, C. The CD94/NKG2C + NK-cell subset on the edge of innate and adaptive immunity to human cytomegalovirus infection. Semin. Immunol. 26, 145-151 (2014).

28. Thapa, M., Kuziel, W.A. \& Carr, D.J. Susceptibility of CCR5-deficient mice to genital herpes simplex virus type 2 is linked to NK cell mobilization. J. Virol. 81, 3704-3713 (2007).

29. van Helden, M.J., Zaiss, D.M. \& Sijts, A.J. CCR2 defines a distinct population of NK cells and mediates their migration during influenza virus infection in mice. PLoS One 7, e52027 (2012).

30. Carr, D.J., Wuest, T. \& Ash, J. An increase in herpes simplex virus type 1 in the anterior segment of the eye is linked to a deficiency in NK cell infiltration in mice deficient in CXCR3. J. Interferon Cytokine Res. 28, 245-251 (2008).

31. Bjorkstrom, N.K. et al. Rapid expansion and long-term persistence of elevated NK cell numbers in humans infected with hantavirus. J. Exp. Med. 208, 13-21 (2011).
32. Petitdemange, C. et al. Unconventional repertoire profile is imprinted during acute chikungunya infection for natural killer cells polarization toward cytotoxicity. PLoS Pathog. 7, e1002268 (2011).

33. Mselle, T.F. et al. Unique characteristics of NK cells throughout the human female reproductive tract. Clin. Immunol. 124, 69-76 (2007).

34. Tomasello, E. et al. Mapping of NKp46(+) cells in healthy human lymphoid and non-lymphoid tissues. Front. Immunol. 3, 344 (2012).

35. Belser, J.A., Rota, P.A. \& Tumpey, T.M. Ocular tropism of respiratory viruses. Microbiol. Mol. Biol. Rev. 77, 144-156 (2013).

36. Daley-Bauer, L.P., Wynn, G.M. \& Mocarski, E.S. Cytomegalovirus impairs antiviral CD8 + T cell immunity by recruiting inflammatory monocytes. Immunity 37, 122-133 (2012).

37. Narayanan, S., Glasser, A., Hu, Y.S. \& McDermott, A.M. The effect of interleukin-1 on cytokine gene expression by human corneal epithelial cells. Exp. Eye Res. 80, 175-183 (2005).

38. Wilson, S.E., Mohan, R.R., Ambrosio, R. Jr, Hong, J. \& Lee, J. The corneal wound healing response: cytokine-mediated interaction of the epithelium, stroma, and inflammatory cells. Prog. Retin. Eye Res. 20, 625-637 (2001).

39. Sakabe, S. et al. Cytokine production by primary human macrophages infected with highly pathogenic H5N1 or pandemic H1N1 2009 influenza viruses. J. Gen. Virol. 92, 1428-1434 (2011).

40. Choy, E.H. \& Panayi, G.S. Cytokine pathways and joint inflammation in rheumatoid arthritis. N. Engl. J. Med. 344, 907-916 (2001).

41. Lee, N. et al. Cytokine response patterns in severe pandemic 2009 H1N1 and seasonal influenza among hospitalized adults. PLoS One 6, e26050 (2011).

42. Gao, R. et al. Cytokine and chemokine profiles in lung tissues from fatal cases of 2009 pandemic influenza A (H1N1): role of the host immune response in pathogenesis. Am. J. Pathol. 183, 1258-1268 (2013).

43. Efron, N., Morgan, P.B. \& Katsara, S.S. Validation of grading scales for contact lens complications. Ophthalmic Physiol. Opt. 21, 17-29 (2001).

44. Shoji, J., Inada, N. \& Sawa, M. Evaluation of eotaxin-1, -2, and -3 protein production and messenger RNA expression in patients with vernal keratoconjunctivitis. Jpn. J. Ophthalmol. 53, 92-99 (2009).

45. Riemens, A., Stoyanova, E., Rothova, A. \& Kuiper, J. Cytokines in tear fluid of patients with ocular graft-versus-host disease after allogeneic stem cell transplantation. Mol. Vis. 18, 797-802 (2012).

46. Fujihara, T. et al. Evaluation of human conjunctival epithelium by a combination of brush cytology and flow cytometry: an approach to the quantitative technique. Diagn. Cytopathol. 17, 456-460 (1997).

47. Martinez-Osorio, $\mathrm{H}$. et al. Characterization and short-term culture of cells recovered from human conjunctival epithelium by minimally invasive means. Mol. Vis. 15, 2185-2195 (2009).

48. Miura-Ochiai, R. et al. Quantitative detection and rapid identification of human adenoviruses. J. Clin. Microbiol. 45, 958-967 (2007).

49. Aoki, K. et al. Epidemic keratoconjunctivitis due to the novel hexonchimeric-intermediate 22,37/H8 human adenovirus. J. Clin. Microbiol. 46, 3259-3269 (2008).

50. Vilches, C., Castano, J., Gomez-Lozano, N. \& Estefania, E. Facilitation of KIR genotyping by a PCR-SSP method that amplifies short DNA fragments. Tissue Antigens 70, 415-422 (2007). 